Virginia Commonwealth University VCU Scholars Compass

2010

\title{
First-principles study of hydrogen adsorption in metal-doped COF-10
}

Miao Miao Wu

Peking University, Virginia Commonwealth University

Qian Wang

Virginia Commonwealth University, qwang@vcu.edu

Qiang Sun

Peking University, Virginia Commonwealth University

Puru Jena

Virginia Commonwealth University, pjena@vcu.edu

Yoshiyuki Kawazoe

Tohoku University

Follow this and additional works at: http://scholarscompass.vcu.edu/phys_pubs

Part of the Physics Commons

Wu, M.M., Wang, Q. Sun, Q. et al. First-principles study of hydrogen adsorption in metal-doped COF-10. The Journal of Chemical Physics, 133, 154706 (2010). Copyright (C) 2010 AIP Publishing LLC.

\section{Downloaded from}

http://scholarscompass.vcu.edu/phys_pubs/111

This Article is brought to you for free and open access by the Dept. of Physics at VCU Scholars Compass. It has been accepted for inclusion in Physics Publications by an authorized administrator of VCU Scholars Compass. For more information, please contact libcompass@vcu.edu. 


\title{
First-principles study of hydrogen adsorption in metal-doped COF-10
}

\author{
Miao Miao Wu, ${ }^{1,2}$ Qian Wang, ${ }^{2, a)}$ Qiang Sun, ${ }^{1,2}$ Puru Jena, ${ }^{2}$ and Yoshiyuki Kawazoe ${ }^{3}$ \\ ${ }^{1}$ Department of Advanced Materials and Nanotechnology and Center for Applied Physics and Technology, \\ Peking University, Beijing 100871, China \\ ${ }^{2}$ Department of Physics, Virginia Commonwealth University, Richmond, Virginia 23284, USA \\ ${ }^{3}$ Institute for Materials Research, Tohoku University, Sendai 980-8577, Japan
}

(Received 17 June 2010; accepted 28 September 2010; published online 21 October 2010)

Covalent organic frameworks (COFs), due to their low-density, high-porosity, and high-stability, have promising applications in gas storage. In this study we have explored the potential of COFs doped with $\mathrm{Li}$ and $\mathrm{Ca}$ metal atoms for storing hydrogen under ambient thermodynamic conditions. Using density functional theory we have performed detailed calculations of the sites $\mathrm{Li}$ and $\mathrm{Ca}$ atoms occupy in COF-10 and their interaction with hydrogen molecules. The binding energy of $\mathrm{Li}$ atom on COF-10 substrate is found to be about $1.0 \mathrm{eV}$ and each $\mathrm{Li}$ atom can adsorb up to three $\mathrm{H}_{2}$ molecules. However, at high concentration, Li atoms cluster and, consequently, their hydrogen storage capacity is reduced due to steric hindrance between $\mathrm{H}_{2}$ molecules. On the other hand, due to charge transfer from $\mathrm{Li}$ to the substrate, $\mathrm{O}$ sites provide additional enhancement for hydrogen adsorption. With increasing concentration of doped metal atoms, the COF-10 substrate provides an additional platform for storing hydrogen. Similar conclusions are reached for Ca doped COF-10. (C) 2010 American Institute of Physics. [doi:10.1063/1.3503654]

\section{INTRODUCTION}

Hydrogen, a clean and "renewable" energy carrier, is considered to play a critical role in a new, decentralized energy infrastructure that can provide power to vehicles, homes, and industries. However, one of the biggest challenges in a new hydrogen economy is finding hydrogen storage materials that must meet stringent requirements for mobile applications, namely, high gravimetric and volumetric density, fast kinetics, and favorable thermodynamics. No materials are available that meet all these requirements simultaneously. In light materials hydrogen is either bound weakly or strongly. In order to have desired kinetics for release, hydrogen stored in quasimolecular form is highly desirable, since the interaction of hydrogen molecule with the substrate is intermediate between van der Waals and chemical interaction. Since there is not enough space in conventional bulk materials to accommodate $\mathrm{H}_{2}$ molecules, high surface area materials like porous materials are hotly pursued. These include metal organic frameworks (MOFs) and covalent organic frameworks (COFs). Due to the large mass of metal atoms such as $\mathrm{Cu}$ and $\mathrm{Zn}$ in MOFs, the gravimetric density for hydrogen storage is quite low. This shortcoming can be avoided in COFs, where the materials are composed of light elements (e.g., C, B, and O) linked by strong covalent bonds. However, the problem is that $\mathrm{H}_{2}$ molecules are only weakly adsorbed on COFs. Pathways to enhance the adsorption while keeping high gravimetric density have become a key issue in materials design for hydrogen storage.

One of the strategies is to dope COFs with light metal atoms. Among these lithium is of special interest, since it is the lightest metal in the periodic table and unlike transition

${ }^{a)}$ Electronic mail: qwang@vcu.edu. metal atoms it does not cluster on carbon fullerenes and nanotubes. In addition, an isolated positive $\mathrm{Li}$ ion can adsorb up to six $\mathrm{H}_{2}$ molecules with adsorption energy that lies between van der Waals and chemical bonding. ${ }^{1-3}$ When Li atoms are deposited on $\mathrm{C}_{60}$ fullerene, charge transfer makes $\mathrm{Li}$ atoms positively charged which then bind hydrogen quasimolecularly. ${ }^{4}$ The binding of hydrogen to $\mathrm{Li}$ can be further improved by doping $\mathrm{C}_{60}$ with B. ${ }^{5}$ These findings have motivated researchers to extend the idea of metal doping to MOFs. ${ }^{6-11}$ It has been found that clustering of transition metal atoms like Sc and Ti on a substrate is one of the key factors affecting the storage performance, ${ }^{12}$ since metal clustering would reduce greatly the amount of stored hydrogen.

$\mathrm{COFs}$ belong to a class of porous polymeric materials with covalent bonds between $\mathrm{C}-\mathrm{C}, \mathrm{C}-\mathrm{O}$, and $\mathrm{B}-\mathrm{O}$. They usually have rigid structure, low densities, and exhibit exceptional thermal stabilities (to temperatures up to $600{ }^{\circ} \mathrm{C}$ ). Their specific surface areas surpass those of well-known zeolites and porous silicates. ${ }^{13}$ Recently, a member of the COF family, COF-10, which is a two-dimensional (2D) mesoporous material, has been synthesized ${ }^{14}$ through cocondensation reactions between 2,3,6,7,10,11-hexahydroxy triphenylene (HHTP) and 4,4'-biphenyldiboronic acid (BPDA). Under appropriate low-pressure regions of the isotherms, it has a surface area of $2080 \mathrm{~m}^{2} \mathrm{~g}^{-1}$, pore volume of $1.44 \mathrm{~cm}^{3} \mathrm{~g}^{-1}$, and one dimensional pore diameter of $34 \AA$. Furukawa and Yaghi carried out an extensive experimental study of storage of hydrogen, methane, and carbon dioxide in the COF materials and found that three-dimensional (3D) COFs (such as COF-102 and COF-103) outperform 2D COFs (COF-1, COF-5, COF-6, COF-8, and COF-10) in their uptake capacities for hydrogen. ${ }^{15}$ More recently, they found that COF-10 shows an exceptional capacity for ammonia. ${ }^{16}$ The simulation of $\mathrm{H}_{2}$ uptake behavior in COF-1 (Ref. 17) 


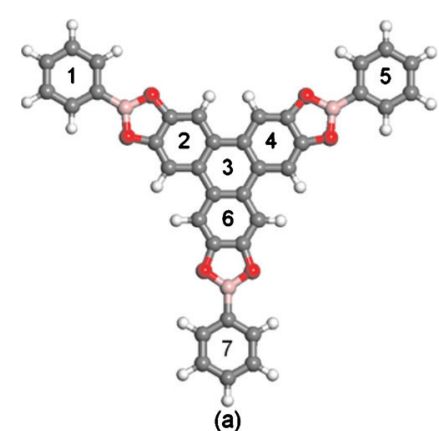

(a)

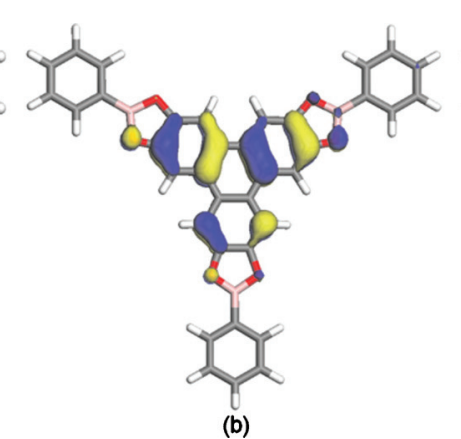

(b)

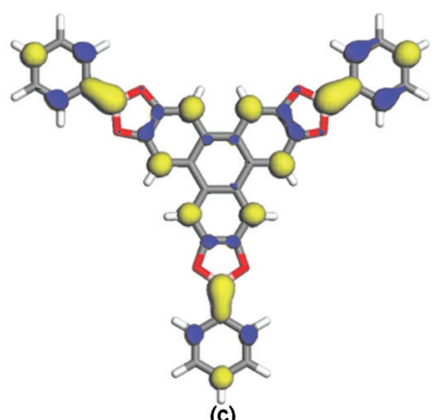

(c)

FIG. 1. (a) Geometry, (b) HOMO, and (c) LUMO of TBE molecule. The sites $1,2, \cdots$ indicate where metal atoms are introduced to study their preferred structure.

and 3D COFs, including COF-102, CoF-103, COF-105, and COF-108, has been performed by several groups. ${ }^{18-21}$ It has been predicted that some COFs can store greater amounts of $\mathrm{H}_{2}$ than MOFs and $\mathrm{Li}$ or $\mathrm{Mg}$ doping can enhance hydrogen adsorption. More interesting, Cao et al. ${ }^{19}$ predicted that $\mathrm{Li}$ doped 3D COFs are the most promising candidates for hydrogen storage to date based on first-principles calculations. However, the theoretical study on pure or metal-decorated COF-10 for hydrogen storage is still lacking. One wonders if this 2D COF material has the potential for hydrogen storage through Li doping and if clustering of the metal atoms is also energetically unfavorable, as is the case in carbon fullerenes ${ }^{4,22}$ and nanotubes. ${ }^{23}$

In this paper, we focused on studying the effect of $\mathrm{Li}$ doping in COF-10 on hydrogen adsorption to search for high capacity COF-based hydrogen storage materials. To this end, some key questions need to be addressed. For example, what sites Li atoms like to occupy? Do they prefer to cluster? Does the metal doping affect the structure and electronic properties of the substrate itself? The answers to these questions are important to better understand and design COFs for hydrogen storage. We note that $\mathrm{Ca}$ also has the capability of enhancing hydrogen adsorption ${ }^{24-27}$ and has a weak tendency of clustering. ${ }^{28}$ Therefore, we have also explored the effect of $\mathrm{Ca}$ doping on the hydrogen storage properties of COF-10.

\section{COMPUTATIONAL PROCEDURE}

The calculations were carried out by using density functional theory (DFT) and generalized gradient approximation (GGA) for exchange-correlation energy. We used the Perdew-Wang 91 form $^{29}$ for GGA and a plane-wave basis set within the projector-augmented-wave (PAW) method originally developed by Blöchl ${ }^{30}$ and adapted by Kresse and Joubert $^{31}$ in the Vienna ab initio simulation package. The particular advantage of the PAW method over the ultrasoft pseudopotentials is that the pseudization of the augmentation charge can be avoided. Though many methods are available for this study, previous benchmark calculations ${ }^{32,33}$ showed that the results using PW91 functional are close to those obtained from MP2 level of theory for describing the noncovalent intermolecular interactions. Some studies ${ }^{34-36}$ have also demonstrated that PW91 is reliable for evaluating the hydrogen adsorption. The structure optimization was symmetry unrestricted and was carried out using conjugategradient algorithm. The convergence for the energy and the force were set to 0.0005 and $0.01 \mathrm{eV} / \AA$, respectively. The energy cutoff was set to $400 \mathrm{eV}$ based on our previous study. ${ }^{12}$ We used a supercell approach where the clusters under investigation were surrounded by $15 \AA$ of vacuum space along the $x, y$, and $z$ directions. Due to the large supercell, the $\Gamma$ point was used to represent the Brillouin zone.

To reduce the computational demand, we have used a fragment of the COF-10, triboronate ester $\left(\mathrm{C}_{36} \mathrm{H}_{21} \mathrm{~B}_{3} \mathrm{O}_{6}\right)$, as shown in Fig. 1. This is labeled as TBE, which is a repeat unit saturated with a hydrogen atom on each end. The structure consists of one HHTP molecule and three fivemembered boronate ester rings $\left(\mathrm{C}_{2} \mathrm{O}_{2} \mathrm{~B}\right)$ connecting three benzene rings. This can be viewed as half of the BPDA molecule with $D_{3 \mathrm{~h}}$ symmetry. The optimized geometry is shown in Fig. 1(a), where we numbered the seven benzene rings for the following discussions. The highest occupied molecular orbital (HOMO) is mainly contributed by the central part in TBE [Fig. 1(b)], while the lowest unoccupied molecular orbital (LUMO) is almost homogeneously distributed over the whole TBE [Fig. 1(c)].

\section{RESULTS AND DISCUSSION}

We began with one Li atom doped in TBE. To determine the preferred site of $\mathrm{Li}$, we have chosen five nonequivalent sites for Li to occupy, thus generating five configurations of Li-TBE. Configurations $I_{1}, I_{2}$, and $I_{3}$ correspond to introducing $\mathrm{Li}$ atom on top of the hollow site of no. 1 benzene ring, $\mathrm{B}-\mathrm{C}$ bridge site and $\mathrm{B}$ on top site next to the ring in Fig. 1(a), respectively. In configurations $I_{4}, \mathrm{Li}$ atom is introduced at top of the hollow site of the five-membered ring of B-O$\mathrm{C}-\mathrm{C}-\mathrm{O}$ between nos. 1 and 2 benzene rings. In configurations $I_{5}$, the Li atom is on the top of the hollow site of no. 2 benzene ring. Optimizations show that the first three initial configurations lead to a stable state, as shown in Fig. 2(a)(1), where the Li atom is on the top hollow site of no. 1 benzene ring. We name it as site- 1 which has a binding energy of 1.05 $\mathrm{eV}$. $\mathrm{Li}-\mathrm{C}$ bond length is found to be $2.20 \AA$. The latter two initial configurations lead to the metastable state. We name the site where $\mathrm{Li}$ atom is on the top hollow site of no. 2 benzene ring as site-2. This site lies $0.2 \mathrm{eV}$ higher in energy than site-1. It is interesting to note that $\mathrm{Li}$ doping has little effect on the HOMO [Fig. 2(b)(1)], but it changes the LUMO substantially [Fig. 2(c)(1)]. The LUMO is highly inhomogeneous and is concentrated on the ring having $\mathrm{Li}$. The HOMO-LUMO gap is calculated to be $0.43 \mathrm{eV}$. 


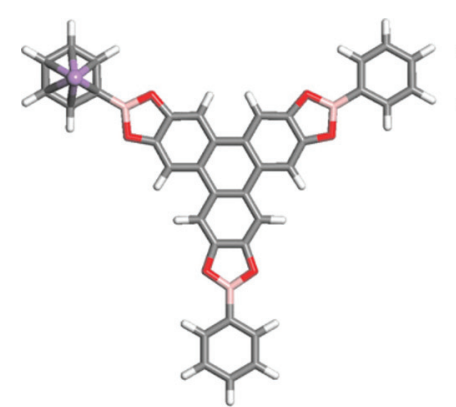

$\left(a_{1}\right)$

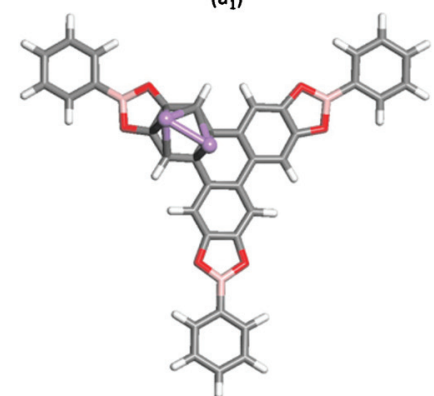

$\left(a_{2}\right)$

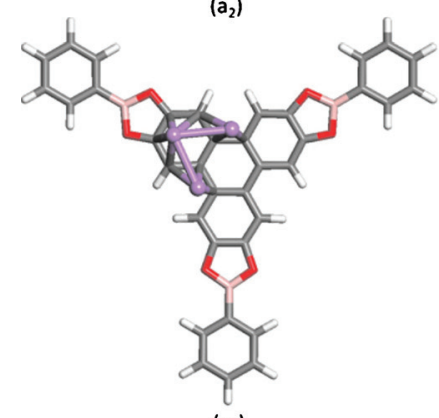

$\left(a_{3}\right)$

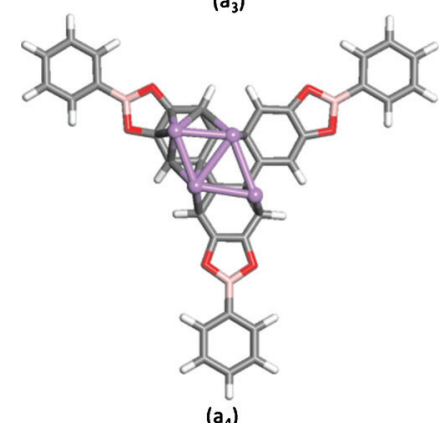

$\left(a_{4}\right)$

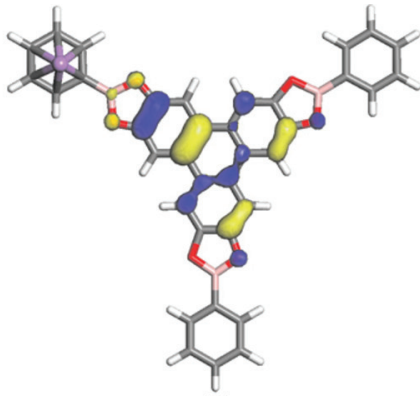

$\left(b_{1}\right)$

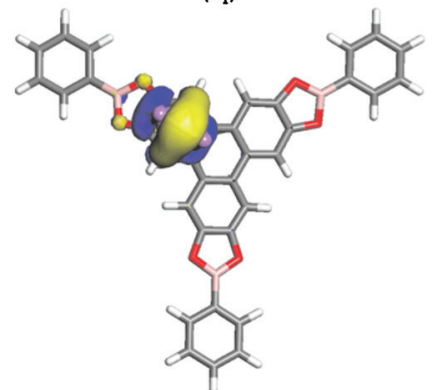

$\left(b_{2}\right)$

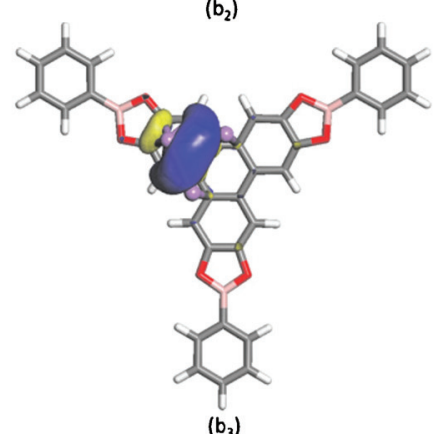

$\left(b_{3}\right)$
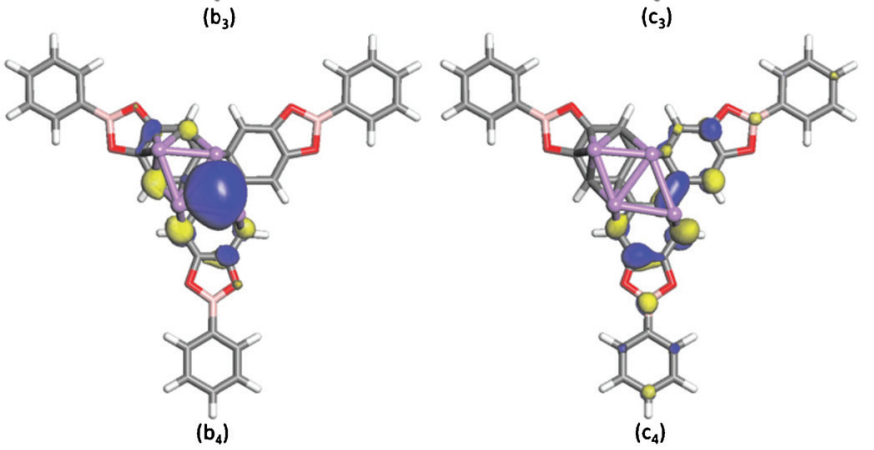

FIG. 2. (a) Geometry, (b) HOMO, and (c) LUMO orbitals for Li-TBE, 2Li-TBE, 3Li-TBE, and 4Li-TBE, respectively. The isosurface value is 0.04 a.u.

We next introduced two lithium atoms to the TBE. There are again many possible sites for two $\mathrm{Li}$ atoms to occupy. We have considered six different initial configurations and only introduced $\mathrm{Li}$ atoms on the top hollow sites of the benzene rings based on our finding as discussed above. The six configurations are labeled as $(1,2),(1,3),(1,5),(1, \overline{2}),(2,3)$, and $(2, \overline{3})$. Here the numbers stand for the benzene rings as shown in Fig. 1(a) and the negative sign denotes $\mathrm{Li}$ atoms occupying the opposite side of the ring. For example, site $(1,2)$ indicates that Li atoms occupy the hollow sites above the rings marked 1 and 2 in Fig. 1 , while site $(1, \overline{2})$ indicates that one $\mathrm{Li}$ atom is above benzene ring 1 while the other is below benzene ring 2 . We found that the lowest energy configuration is $(2,3)$. Here the two $\mathrm{Li}$ atoms form a dimer with the $\mathrm{Li}-\mathrm{Li}$ bond length of $2.43 \AA$. They reside on the top of no. 2 benzene ring, as shown in Fig. 2(a)(2). The binding energy for each $\mathrm{Li}$ atom is $1.11 \mathrm{eV}$ and the average $\mathrm{Li}-\mathrm{C}$ bond length is $2.18 \AA$. In Table I we give the relative energy for all the configurations considered. The Li dimer caused a local distortion in geometry and the structure was bent outward. The HOMO is mainly on Li dimer [Fig. 2(b)(2)], while the LUMO is on other rings [Fig. 2(c)(2)].

To find the preferred configuration when three $\mathrm{Li}$ atoms are introduced, we considered five configurations labeled $(1,2,3),(1,3,5),(1,5,7),(2,3,4)$, and $(2,4,6)$. The configuration of $(2,4,6)$ gave the lowest energy, approximately 0.21 $\sim 0.44 \mathrm{eV}$ lower in energy than the others (Table I). In the lowest energy configuration, the three $\mathrm{Li}$ atoms move together forming an isosceles triangle with $\mathrm{Li}-\mathrm{Li}$ bond lengths of 2.77, 2.77, and $3.21 \AA$, as shown in Fig. 2(a)(3). This 
TABLE I. The relative energy $(\Delta E)$ (in eV) and the binding energy per Li atom (BE/Li) (in eV) for doping two, three, and four Li atoms at different sites on TBE. The numbering of sites for initial configurations (Config.) is explained in the text. For example, $(1,2)$ configuration indicates that two Li atoms were introduced at sites 1 and 2 in Fig. 1.

\begin{tabular}{|c|c|c|c|c|c|c|c|c|c|c|c|}
\hline \multirow[b]{2}{*}{ Config. } & \multicolumn{2}{|c|}{ 1Li-TBE } & \multirow[b]{2}{*}{ Config. } & \multicolumn{2}{|c|}{ 2Li-TBE } & \multirow[b]{2}{*}{ Config. } & \multicolumn{2}{|c|}{ 3Li-TBE } & \multirow[b]{2}{*}{ Config. } & \multicolumn{2}{|c|}{ 4Li-TBE } \\
\hline & $\Delta E$ & $\mathrm{BE} / \mathrm{Li}$ & & $\Delta E$ & $\mathrm{BE} / \mathrm{Li}$ & & $\Delta E$ & $\mathrm{BE} / \mathrm{Li}$ & & $\Delta E$ & $\mathrm{BE} / \mathrm{Li}$ \\
\hline (Site-1) & 0.00 & 1.05 & $(1,2)$ & 0.05 & 1.09 & $(1,2,3)$ & 0.38 & 1.02 & $(1,3,5,7)$ & 0.66 & 1.06 \\
\hline \multirow[t]{5}{*}{ (Site-2) } & 0.20 & 0.85 & $(1,3)$ & 0.30 & 0.96 & $(1,3,5)$ & 0.21 & 1.08 & $(1, \overline{3}, 5,7)$ & 0.64 & 1.07 \\
\hline & & & $(1,5)$ & 0.15 & 1.04 & $(1,5,7)$ & 0.32 & 1.04 & $(2,3,4,6)$ & 0.65 & 1.06 \\
\hline & & & $(1, \overline{2})$ & 0.29 & 0.97 & $(2,3,4)$ & 0.44 & 1.00 & $(2, \overline{3}, 4,6)$ & 0.51 & 1.10 \\
\hline & & & $(2,3)$ & 0.00 & 1.11 & $(2,4,6)$ & 0.00 & 1.15 & (Rhomb) & 0.00 & 1.23 \\
\hline & & & $(2, \overline{3})$ & 0.26 & 0.98 & & & & (Tetrahedron) & 0.04 & 1.22 \\
\hline
\end{tabular}

geometry can be considered as adding one more $\mathrm{Li}$ atom to the 2Li-TBE structure, but unlike the 2Li-TBE, the introduction of three $\mathrm{Li}$ atoms does not cause much distortion in geometry. And in this case, the HOMO and LUMO are on the $\mathrm{Li}_{3}$ cluster [see Fig. 2(b)(3) and Fig. 2(c)(3)].

To find the equilibrium structure of four $\mathrm{Li}$ atoms, we studied six initial configurations. The first four are labeled $(1,3,5,7),(1, \overline{3}, 5,7),(2,3,4,6)$, and $(2, \overline{3}, 4,6)$. The fifth and the sixth configurations are based on the $3 \mathrm{Li}-\mathrm{TBE}$ by adding one more Li either forming a rhombus or a tetrahedron, respectively. The structure optimizations indicate that the rhombus configuration has the lowest energy while the tetrahedron is only $0.04 \mathrm{eV}$ higher in energy. The remaining four configurations are much higher in energy with the energy difference lying in the range of $0.51-0.66 \mathrm{eV}$. The HOMO is mainly on Li atoms, while the LUMO is mainly on $\mathrm{C}$ sites next to the $\mathrm{Li}_{4}$ cluster. This is different from other cases we discussed above. It is clear that $\mathrm{Li}$ atoms prefer to cluster, which has been further confirmed by adding more Li atom to the TBE. For instance, the clustering configuration of seven $\mathrm{Li}$ on the TBE with compact $C_{3 \mathrm{~V}}$ structure is found to be $0.78 \mathrm{eV}$ lower in energy than the one with one $\mathrm{Li}$ on each top hollow site of the seven numbered benzene rings in Fig. 1(a).

Next we discuss hydrogen adsorption. One of the quantities we used to measure the thermodynamics is adsorption energy (AE), which is defined as the energy difference between the complex and its fragments,

$$
\begin{aligned}
\mathrm{AE}\left(\mathrm{H}_{2}\right)= & -\left[E\left(\mathrm{TBE}+m \mathrm{Li}+n \mathrm{H}_{2}\right)-E(\mathrm{TBE}+m \mathrm{Li})\right. \\
& \left.-E\left(n \mathrm{H}_{2}\right)\right] / n
\end{aligned}
$$

Here $m$ is the number of $\mathrm{Li}$ atoms and $n$ is the number of hydrogen molecules. For Li-TBE, when one $\mathrm{H}_{2}$ molecule is introduced to $\mathrm{Li}$ site, the equilibrium distance $\mathrm{H}_{2}$ and $\mathrm{Li}$ site is $2.13 \AA$, and the bond length of $\mathrm{H}_{2}$ is elongated to $0.76 \AA$. The adsorption energy is found to be $0.12 \mathrm{eV}$. When two and three $\mathrm{H}_{2}$ molecules are trapped, the distance to Li changes to 2.18 and $2.23 \AA$, and the adsorption energy is slightly reduced to $0.11 \mathrm{eV}$. In $4 \mathrm{Li}-\mathrm{TBE}$, four $\mathrm{Li}$ atoms form a rhombus, with each Li site trapping one $\mathrm{H}_{2}$ molecule [Fig. 3(a)]; the average adsorption energy is $0.14 \mathrm{eV} / \mathrm{H}_{2}$. When two $\mathrm{H}_{2}$ molecules are initially introduced to each Li site, the structure optimization shows that only six of them can be trapped due to the steric hindrance [Fig. 3(b)]. The average adsorption energy is found to be $0.15 \mathrm{eV} / \mathrm{H}_{2}$, which is larger than that for $4 \mathrm{Li}-\mathrm{TBE}-4 \mathrm{H}_{2}$. The distance to $\mathrm{Li}$ also increases to $2.33 \AA$ (Table II). These changes cannot be understood based just on the polarizing interactions of $\mathrm{Li}$ ions. In fact in this case, some $\mathrm{H}_{2}$ molecules are closer to the charged $\mathrm{O}$ sites and the added interaction with $\mathrm{O}$ sites increases the adsorption energy. Thus, we see that metal doping, due to charge transfer to the substrate, cannot only make it become the trap site but also additional adsorption induced on the substrate itself can enhance adsorption energy. Therefore, as the doping concentration increases the geometry and the interactions become complicated. There are two competing factors associated with high doping concentration; clustering of Li atoms reduces the available $\mathrm{Li}$ site for hydrogen adsorption while additional adsorption induced by the substrate can bind hy-
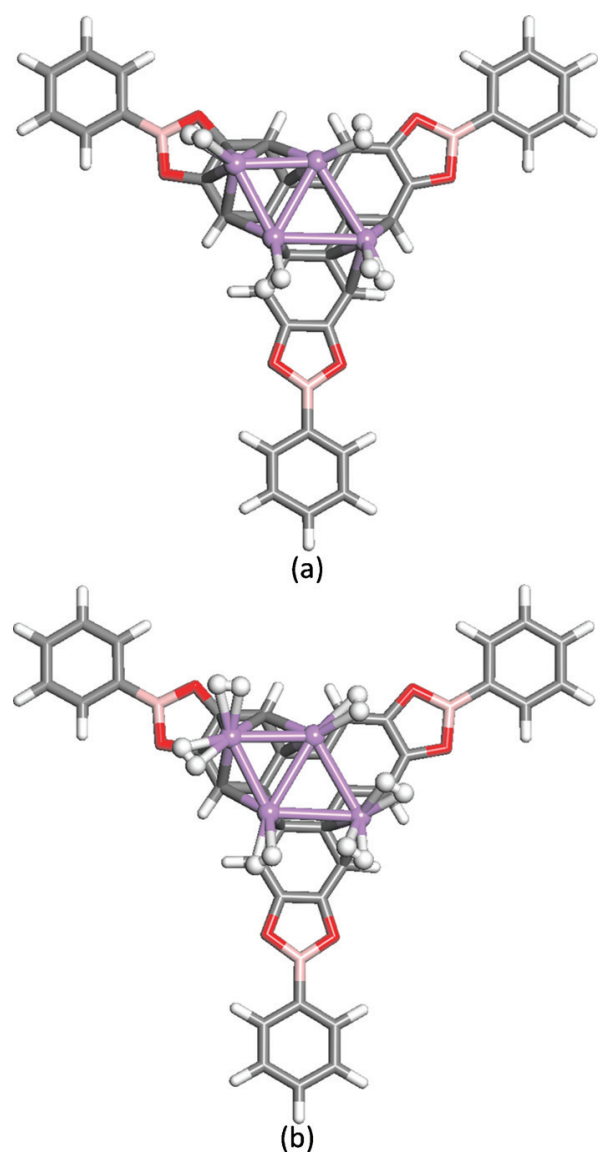

FIG. 3. Optimized geometry of (a) $4 \mathrm{Li}-\mathrm{TBE}-4 \mathrm{H}_{2}$ and (b) $4 \mathrm{Li}-\mathrm{TBE}-6 \mathrm{H}_{2}$. 
TABLE II. The adsorption energy per $\mathrm{H}_{2}\left(\mathrm{AE} / \mathrm{H}_{2}\right)$ (in eV), the average bond lengths for $\mathrm{C}-\mathrm{Li}, \mathrm{Li}-\mathrm{H}$, and $\mathrm{H}-\mathrm{H}$ bonds (in angstrom), and the energy gaps (in $\mathrm{eV}$ ) of TBE doped with one $\mathrm{Li}$ and four $\mathrm{Li}$ atoms, respectively.

\begin{tabular}{lccccc}
\hline \hline Clusters & $\mathrm{AE} / \mathrm{H}_{2}$ & $\mathrm{C}-\mathrm{Li}$ & $\mathrm{Li}-\mathrm{H}$ & $\mathrm{H}-\mathrm{H}$ & Gap \\
\hline $\mathrm{Li}-\mathrm{TBE}+1 \mathrm{H}_{2}$ & 0.12 & 2.21 & 2.13 & 0.76 & 0.37 \\
$\mathrm{Li}-\mathrm{TBE}+2 \mathrm{H}_{2}$ & 0.11 & 2.23 & 2.18 & 0.76 & 0.39 \\
$\mathrm{Li}-\mathrm{TBE}+3 \mathrm{H}_{2}$ & 0.11 & 2.26 & 2.23 & 0.76 & 0.46 \\
$4 \mathrm{Li}-\mathrm{TBE}+4 \mathrm{H}_{2}$ & 0.13 & 2.22 & 1.98 & 0.76 & 0.64 \\
$4 \mathrm{Li}-\mathrm{TBE}+6 \mathrm{H}_{2}$ & 0.15 & 2.33 & 1.97 & 0.77 & 0.76 \\
\hline \hline
\end{tabular}

drogen on the substrate. In our calculations we have not included zero-point energies (ZPEs) or thermal corrections. Previous studies have found that the $\mathrm{ZPE}$ of $\mathrm{H}_{2}$ in porous materials lies in the range of $0.01-0.02 \mathrm{eV} / \mathrm{H}_{2} \cdot{ }^{37-39}$ Based on these results, we feel that ZPE corrections will not have significant effect on our calculated adsorption energy. Thermal energy corrections at room temperature are of the order of $\mathrm{meV}$ and the adsorption energy would lie in the range of $0.08-0.12 \mathrm{eV}$, which is close to the energy window of $0.1-0.2 \mathrm{eV} / \mathrm{H}_{2}$ required for applications under ambient thermodynamic conditions. 40

We note that the Li-doped 2D COF-10 is unlike the 3D $\mathrm{COFs}^{18,19}$ due to their spatial structures and chemical constitutions. Although the average binding energies per $\mathrm{Li}$ in both systems are comparable (namely, 1.05, 1.11, 1.15, and 1.23 $\mathrm{eV} / \mathrm{Li}$ for one to four $\mathrm{Li}$ atoms on the TBE of COF-10 versus $1.08 \mathrm{eV} / \mathrm{Li}$ for eight $\mathrm{Li}$ atoms on the TBPM of 3D COFs),${ }^{19}$ the hydrogen adsorption energy of the Li-doped COF-10 is smaller than that of the Li-doped 3D COFs. For instance, the adsorption energy of the Li ion-doped 3D COFs was found to be $0.28 \mathrm{eV} / \mathrm{H}_{2},{ }^{18}$ while that for the Li-doped COF-10 is in the range of $0.11-0.15 \mathrm{eV}$. We recall from the case of $\mathrm{Li}_{12} \mathrm{C}_{60}$ (Ref. 4) that due to the special geometry of the fullerene, $12 \mathrm{Li}$ atoms are separately capped on 12 pentagons of $\mathrm{C}_{60}$ and each $\mathrm{Li}$ site has enough space to trap hydrogen molecules. However, $\mathrm{Li}$ atoms on COF-10 tend to cluster. We also recall that $\mathrm{C}_{60}$ uniformly coated with a layer of $\mathrm{Ca}$ has been shown to be an effective trap of hydrogen. ${ }^{23}$ Therefore, we explored the possibility of Ca doping to enhance the capacity of hydrogen storage in COF-10

Following similar procedure described above, we have further studied $\mathrm{Ca}$ doping. We found that $\mathrm{Ca}$ atoms also have the tendency to cluster in COF-10. For this study we introduced four $\mathrm{Ca}$ onto the TBE substrate. Four different initial configurations with $\mathrm{Ca}$ atoms occupying $(1,3,5,7)$ and
$(1, \overline{3}, 5,7)$ sites in Fig. 1 and forming both planar rhombus and tetrahedral geometries were considered. Analogous to the case of 4Li-TBE structure, the lowest energy configuration in the $\mathrm{Ca}$ doped case is the one where four $\mathrm{Ca}$ atoms form a rhombus cluster. This is about $0.80 \mathrm{eV}$ lower in energy than that of $(1,3,5,7)$ site. The relaxed structure and the HOMO and LUMO orbitals for the lowest energy configuration are plotted in Fig. 4. They show that most of the frontier orbitals for both the HOMO and LUMO are mainly focused on $\mathrm{Ca}$ atoms, while the LUMO of $4 \mathrm{Li}-\mathrm{TBE}$ is mainly located on $\mathrm{C}$ sites near to the $\mathrm{Li}_{4}$ cluster. Compared with $\mathrm{Li}$, more charge is transferred to the substrate due to the higher valency of $\mathrm{Ca}$. Therefore, $\mathrm{Ca}$ atoms carry larger positive charge than $\mathrm{Li}$. This results in a stronger local electric field, larger polarization of the $\mathrm{H}_{2}$ molecules, and higher binding energy of $\mathrm{H}_{2}$. The adsorption energy is 0.21 and $0.16 \mathrm{eV} / \mathrm{H}_{2}$ for Ca-TBE- $\mathrm{H}_{2}$ and $4 \mathrm{Ca}-\mathrm{TBE}-4 \mathrm{H}_{2}$ respectively. Therefore, $\mathrm{Ca}$ doping provides a better platform for enhancing hydrogen adsorption thermodynamics in COF-10.

\section{CONCLUSIONS}

In summary, we have studied the potential of metaldoped COF-10 as hydrogen storage materials. We doped COF-10 with up to four $\mathrm{Li}$ and $\mathrm{Ca}$ atoms. We then studied the interaction of these doped metal atoms with up to three hydrogen molecules. Charge transfer from the metal atoms to COF-10 leaves the metal atoms in a positively charged state and hydrogen atoms are bound to these sites in quasimolecular form through the polarization mechanism. ${ }^{1}$ However, unlike in the case of $\mathrm{Li}$ and $\mathrm{Ca}$ doped $\mathrm{C}_{60}$, these metal atoms were found to cluster on the COF-10 substrate, the clustering of metal atoms in COF-10 not only slightly distorts the substrate outwardly but also reduces the available sites for hydrogen to be adsorbed. On the other hand, the charge induced

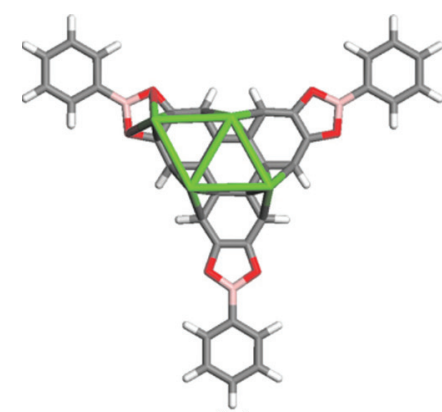

(a)

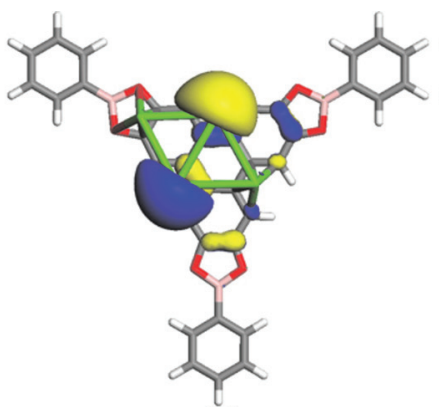

(b)

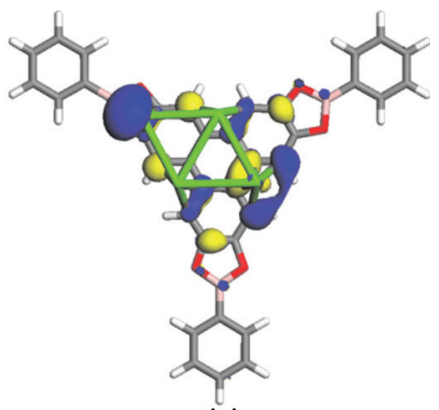

(c)

FIG. 4. (a) Geometry, (b) HOMO, and (c) LUMO orbitals for 4Ca-TBE. 
on the COF-10 substrate helps to increase the adsorption energy. The frontier orbitals of COF-10 can be tuned by changing the doping concentration. As concentration of metal atoms increases, the $\mathrm{COF}-10$ substrate becomes a more effective trap of hydrogen. These two competing factors show that metal-doped COF-10 could be a potential candidate for hydrogen adsorption.

\section{ACKNOWLEDGMENTS}

This work is partially supported by grants from the U.S. Department of Energy, the National Natural Science Foundation of China (NSFC-20973010, NSFC-10874007), and the China Scholarship Council. The authors thank the crew of the Center for Computational Materials Science, the Institute for Materials Research, Tohoku University (Japan), for their continuous support of the HITACH SR11000 supercomputing facility.

${ }^{1}$ J. Niu, B. K. Rao, and P. Jena, Phys. Rev. Lett. 68, 2277 (1992).

${ }^{2}$ B. K. Rao and P. Jena, Europhys. Lett. 20, 307 (1992).

${ }^{3}$ J. Niu, J. B. K. Rao, P. Jena, and M. Manninen, Phys. Rev. B 51, 4475 (1995).

${ }^{4}$ Q. Sun, P. Jena, Q. Wang, and M. Marquez, J. Am. Chem. Soc. 128, 9741 (2006)

${ }^{5}$ Q. Sun, Q. Wang, and P. Jena, Appl. Phys. Lett. 94, 013111 (2009).

${ }^{6}$ S. Yang, X. Lin, A. J. Blake, G. S. Walker, P. Hubberstey, N. R. Champness, and M. Schroder, Nat. Chem. 1, 487 (2009).

${ }^{7}$ G. de Combarieu, S. Hamelet, F. Millange, M. Morcrette, M. Tarascon, G. Ferey, and R. Walton, Electrochem. Commun. 11, 1881 (2009).

${ }^{8}$ D. Banerjee, S. J. Kim, and J. B. Parise, Cryst. Growth Des. 9, 2500 (2009)

${ }^{9}$ T. Wu, J. Zhang, X. Bu, and P. Feng, Chem. Mater. 21, 3830 (2009).

${ }^{10}$ E. Klontzas, A. Mavrandonakis, E. Tylianakis, and G. E. Froudakis, Nano Lett. 8, 1572 (2008).

${ }^{11}$ A. Mavrandonakis, E. Tylianakis, A. K. Stubos, and G. E. Froudakis, J. Phys. Chem. C 112, 7290 (2008).

${ }^{12}$ Q. Sun, Q. Wang, P. Jena, and Y. Kawazoe, J. Am. Chem. Soc. 127, 14582 (2005).

${ }^{13}$ A. P. Côté, A. I. Benin, N. W. Ockwig, M. O'Keeffe, A. J. Matzger, and O. M. Yaghi, Science 310, 1166 (2005).
${ }^{14}$ A. P. Côté, H. M. El-Kaderi, H. Furukawa, J. R. Hunt, and O. M. Yaghi, J. Am. Chem. Soc. 129, 12914 (2007).

${ }^{15}$ H. Furukawa and O. M. Yaghi, J. Am. Chem. Soc. 131, 8875 (2009).

${ }^{16}$ C. J. Doonan, D. J. Tranchemontagne, T. G. Glover, J. R. Hunt, and O. M. Yaghi, Nat. Chem. 2, 235 (2010)

${ }^{17}$ P. Srepusharawoot, R. H. Scheicher, C. M. Araújo, A. Blomqvist, U. Pinsook, and R. Ahuja, J. Phys. Chem. C 113, 8498 (2009).

${ }^{18}$ Y. Choi, J. Lee, J. Choi, and J. Kang, Appl. Phys. Lett. 92, 173102 (2008).

${ }^{19}$ D. Cao, J. Lan, W. Wang, and B. Smit, Angew. Chem., Int. Ed. 48, 4730 (2009).

${ }^{20}$ E. Klontzas, E. Tylianakis, and G. Froudakis, J. Phys. Chem. C 113, 21253 (2009).

${ }^{21}$ J. Lan, D. Cao, and W. Wang, J. Phys. Chem. C 114, 3108 (2010).

${ }^{22}$ F. Rabilloud, J. Phys. Chem. A 114, 7241 (2010).

${ }^{23}$ I. Cabria, M. J. López, and J. A. Alonso, J. Chem. Phys. 123, 204721 (2005).

${ }^{24}$ Q. Wang, Q. Sun, P. Jena, and Y. Kawazoe, J. Chem. Theory Comput. 5, 374 (2009).

${ }^{25}$ C. Ataca, E. Akturk, and S. Ciraci, Phys. Rev. B 79, 041406 (2009).

${ }^{26}$ X. Yang, R. Q. Zhang, and J. Ni, Phys. Rev. B 79, 075431 (2009).

${ }^{27}$ Y. Y. Sun, K. Lee, Y. H. Kim, and S. B. Zhang, Appl. Phys. Lett. 95, 033109 (2009).

${ }^{28}$ J. W. Mirick, C. H. Chien, and E. Blaisten-Barojas, Phys. Rev. A 63, 023202 (2001).

${ }^{29}$ Y. Wang and J. P. Perdew, Phys. Rev. B 44, 13298 (1991).

${ }^{30}$ P. E. Blöchl, Phys. Rev. B 50, 17953 (1994).

${ }^{31}$ G. Kresse and J. Joubert, Phys. Rev. B 59, 1758 (1999).

${ }^{32}$ C. Enkvist, Y. Zhang, and W. Yang, Int. J. Quantum Chem. 79, 325 (2000).

${ }^{33}$ S. Tsuzuki and H. P. Lüthi, J. Chem. Phys. 114, 3949 (2001).

${ }^{34}$ Y. Y. Sun, K. Lee, L. Wang, Y. H. Kim, W. Chen, Z. Chen, and S. B. Zhang, Phys. Rev. B 82, 073401 (2010).

${ }^{35} \mathrm{~F}$. Li, J. Zhao, B. Johansson, and L. Sun, Int. J. Hydrogen Energy 35, 266 (2010).

${ }^{36}$ Z. Zhou, J. Zhao, Z. Chen, X. Gao, T. Yan, B. Wen, and P. Schleyer, J. Phys. Chem. B 110, 13363 (2006).

${ }^{37}$ S. A. FitzGerald, J. Hopkins, B. Burkholder, and M. Friedman, Phys. Rev. B 81, 104305 (2010).

${ }^{38}$ L. Kong, G. Román-Pérez, J. M. Soler, and D. C. Langreth, Phys. Rev. Lett. 103, 096103 (2009).

${ }^{39}$ K. Sillar, A. Hofmann, and J. Sauer, J. Am. Chem. Soc. 131, 4143 (2009).

${ }^{40}$ S. K. Bhatia and A. L. Myers, Langmuir 22, 1688 (2006). 